\title{
Parameters Affecting Underwater Channel Communication Performance
}

\author{
Sheeraz Ahmed ${ }^{1}$, Malik Taimur $\mathrm{Ali}^{2}$ \\ Department of Computer Science \\ Iqra National University, Peshawar, Pakistan \\ Saqib Shahid Rahim ${ }^{3}$ \\ Department of Computer Science \\ Abasyn University, Peshawar, Pakistan
}

\author{
Zahid Farid $^{4}$ \\ University of Science and Technology \\ Bannu, Pakistan
}

Owais Amanullah Khan
Career Dynamics Research Center
Peshawar, Pakistan

\author{
Zeeshan Najam ${ }^{6}$ \\ Ultimate Consultancy, Peshawar, Pakistan
}

\begin{abstract}
Underwater or Acoustic propagation is characterized by three major factors: attenuation that increases with signal frequency, time-varying multipath propagation, and low speed of sound. The background noise, often characterized as Gaussian, is not white, but has a decaying power spectral density. Channel capacity depends on the distance, and may be extremely limited. As acoustic propagation is best supported at low frequencies, an acoustic communication system is inherently wideband and bandwidth is not negligible with respect to its center frequency. The channel has a sparse impulse response, where each physical path acts as a time-varying low-pass filter, and motion introduces Doppler spreading and shifting. Surface waves, internal turbulence and fluctuations in sound speed, contribute to random signal variations. Till date, there are no standardized models for acoustic channel fading. Experimental measurements are often made to assess the statistical properties of the underwater channel.
\end{abstract}

\section{Keywords-UWSN; doppler effect; attenuation; noise; salinity}

\section{INTRODUCTION}

Electromagnetic signals that move from source to destination generally come across with various hindrances. The various models that governs the transmission of signals; focus on how all these factors affect transmission. Figure 1 depicts the basic structure of transmission system. It consists of three components that are 'Sender', 'Channel' or 'Medium' and 'Receiver'.

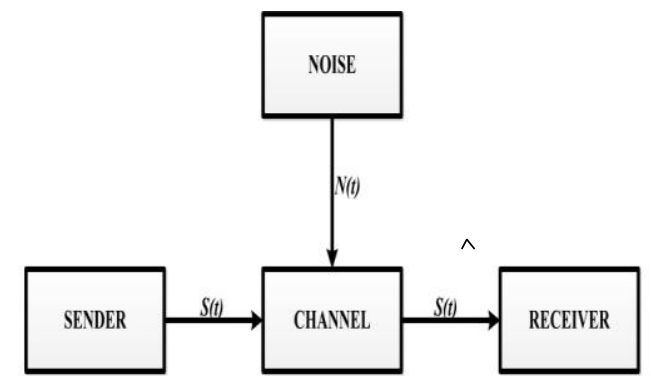

Fig. 1. Basic Communication Model.
Where $s(t)$ is the signal that is transmitted to channel, $\hat{s}(t)$ is the signal that is been received and $N(t)$ are the noises that are affecting the channel. For underwater, acoustic waves are used for communication channels. The acoustic channels are among the critical and challenging medium in recent research [1]. Its features include long propagation and attenuation which is frequency dependent that are extremely affected by the link orientation and by nodes distance [2].

The acoustic links are mostly affected by multi path, Doppler spread, path loss, variable propagation delay and different noises [3].

At low frequencies the propagation of acoustic signals is outstanding however, for the transmission channel its capacity is very least [1]. The range of frequency for acoustic wave is from 10 to 15 kilo Hertz while channel capacity is just 5kilo Hertz and its speed is nearly 1500 meter per second. The propagation is over several channels. High Doppler Effect arises due to mobility of nodes [2]. In fact multi-path propagation, low speed of sound and frequency-dependent attenuation results in Doppler Effect that makes the acoustic channel a challenging medium.

Instead of acoustic waves for underwater the radio signals are not properly propagated apart from low frequencies for very small distances [4]. Similarly the optical waves that are well applied in blue-green area about $500 \mathrm{~nm}$ suffer too by attenuation and do not propagate greater than $100 \mathrm{~m}$ even it have channel capacity in MHz order [5]. Hence, the acoustic signals are considered best for this communication however, it covers a small range. These waves can propagate over kilometers or hundreds of kilo-meters, but for the coverage of longer distance, lower frequency is required [6].

Underwater (UW) acoustic transmission over very larger scales that is in thousands $\mathrm{km}$ can be possible in one hop but the channel capacity must be in order of 10 Hertz [7]. The horizontal channel is extremely difficult for the multi-path 
propagation. However, the vertical transmission shows minimum distortion [8].

Concept of UWSN of distributed autonomous motes [9] is presented by improvements and establishments in sensor technology, vehicle and acoustic modems technology. Currently research is ongoing for standardizing UWSN schemes to approve interoperability of various modems. An international NATO led is operational on formation Janus which is an international standard to specify structure of packet as well as rate of bit mechanisms of acknowledgement, etc. for applying in a given frequency distance configurations [10].

Due to lack of "typical applications," two kinds of networks are possible. The first is that have static motes and deployment of these are for lengthy time and these networks are designed for the monitoring of environment. The other one is the one that have not fixed nodes and they are not deployed for longer time. These types of networks are planned for fleets of cooperating AUVs, where vehicles can make decisions by responding to each other but the most significant aspect is energy efficiency for bottom mounted networks.

This article is organized into four sections. Section II provides some existing work. Section III that is further divided in to different parameters of propagation channel that are attenuation, noise, Signal-to-Noise Ratio (SNR), bandwidth, multipath propagation, Delay spread, Doppler distortion, Fading and Scattering are discussed. Finally the article is settled in section IV to consider the effect on upcoming UWSN schemes.

\section{LITERATURE REVIEW}

At the end of World War II United States developed initial acoustic communication system for underwater. In that system the 8 to $11 \mathrm{kHz}$ band with analog modulation used [11]. But with the passage of time the technology is improved and digital modulation mechanisms came in use. In the era of 80's researchers of Massachusetts Institute of Technology proposed a new system; Frequency Shift Keying (FSK) [12]. It becomes the foundation of commercial base digital acoustic modems of 1st generation [13]. After FSK different acoustic modems developed that based on coded FSK such as WHOI micro modem [14]. As FSK depends on non-coherent detection hence it provides strength to channel losses but its utilization of bandwidth not so well. The research of 90s emphasis on quadrature amplitude modulation and phase shift keying for underwater acoustic channels which provides additional bits per second per hertz of employed channel capacity. Northeastern University and WHOI work for improvements and enhancements which come out with a channel equalization or synchronization method [15]. These efforts become the base of high speed acoustic modem 2nd generation.

The authors of [16] examined acoustic channel features such as noise, speed of sound (propagation speed) with variations in frequency, attenuation, salinity, depth and temperature through some models and mathematical expressions for the area of UWSNs. According to Cooperative Energy-Efficient Protocol for UWSN (Co-UWSN) [17] the researchers used cooperation approach to maximize lifetime of the UWSN, increase the Packet Delivery Ratio (PDR) using SNR and declined consumptions of energy that is mainly advantageous for time-critical as well as delay-sensitive applications. It alleviates possessions of multipath fading and noise using cooperative base transmissions of data. The number of qualified neighbors rises by changing in depth threshold; hence in delay-sensitive applications the acute data loss is minimized. The cooperation character and the optimal weight calculation deliver complementary of load for UWSN with stability of UWSN is heightened too.

The scheme Stochastic Performance Analysis with Reliability and Cooperation (SPARCO) [18] take the features of cooperation approach for increasing and improving the performance of UWSN. Cooperation is utilized for developing a routing technique that is efficient in terms of consumption of energy. The sensors of UWSN are assumed to be entailing of a uni-directional antenna. To reduce the energy usage, multi nodes send their data packets cooperation based by taking the benefits of spatial diversity. Techniques that comprise single hop or greater than one both exploits to declining path losing exist in links connecting sensor nodes and then forwards the data packet.

\section{PRopagation CHANNEl PARAMETERS}

This article is an overview of the acoustic channel features whose purpose is to expose those aspects of propagation that are significant for the design of acoustic communications. Following are the parameters of UW propagation channel.

\section{A. Attenuation}

A unique characteristic of an acoustic link is the path loss based on frequency of the signal. The signal dispersion loss and absorption loss are maximized by maximizing distance. Following expression is for the net path loss [19]

$A(d, f)=\left(d / d_{r e f}\right)^{k} a(f)^{d-d_{r e f}}$

In equation $1 \mathrm{f}$ is the signal frequency while 1 is distance of transmission which taken in reference to $d_{\text {ref }}$. The exponent $k$ models loss of spreading. Its typical values lie between 1 and 2 for cylindrical and spherical spreading respectively. The coefficient of absorption modeled empirically by Thorp's formula which gives $\boldsymbol{a}(\boldsymbol{f})$ in $\mathrm{dB} / \mathrm{km}$ for $\boldsymbol{f}$ in $\mathrm{kHz}$ as [18].

$10 \log a(f)=0.11 \frac{f^{2}}{1+f^{2}}+44 \frac{f^{2}}{4100+f^{2}}+2.75 \times 10^{-4} f^{2}+$
0.003

(for $f>0.4$ ) $[\mathrm{dB} / \mathrm{km}]$

$10 \log a(f)=0.002+\frac{0.11 f}{1+f}+0.011 f$

(for $f<0.4) \quad[\mathrm{dB} / \mathrm{km}]$

This function is accurate for frequencies that are exceeding a few hundred $\mathrm{Hz}$. It neglects the effect of frequencies caused by boric acid and magnesium sulfate, salinity and acidity levels of the water in sea or ocean, so they may not leads to very accurate result [16]. 


\section{B. Noise}

In acoustic channel site specific and ambient noises exists. The first one presents specific sites while in second one noise always exists in background of underwater. It arises by distant shipping, turbulence, breaking waves and thermal noise. The turbulence noise is represented by $\left(N_{t}\right)$, shipping noise by $\left(N_{s}\right)$, waves noise by $\left(N_{\omega}\right)$, and thermal noise by $\left(N_{t h}\right)$. The Power Spectral Density (PSD) of $N_{t}, N_{s}, N_{\omega}, N_{t h}$ are modeled in $\mathrm{dB}$ re $\mu \mathrm{Pa} / \mathrm{Hz}$ as frequency function in kilo $\mathrm{Hz}$.

1) Turbulence noise: Turbulence associated with surface disturbance or tidal flow around an obstruction generates continuous noise. Turbulence is also caused by storms or during the rain events. It may be produced by marine life. The turbulence noise is formulated as

$10 \log N_{t}(f)=17-30 \log f$

2) Shipping noise: Another type of noise is the one caused by ship traffic. The effect of ship traffic is concerned with the number of ships and the distance of shipping from the area of study. The shipping noise is formulated as

$10 \log N_{s}(f)=40+20(s-0.5)+26 \log f-60 \log (f+$

with $\mathrm{s}$ as the shipping factor which lies between 0 and 1 for low and high activities respectively.

3) Wave noise: Wave noise is caused due to the movement of waves in the sea or ocean. The wave noise formulated as

$10 \log N_{\omega}(f)=50+7.5 \omega^{1 / 2}+20 \log f-40 \log (f+0.4)$

with $\omega$ as the wind speed in $\mathrm{m} / \mathrm{s}$. The movement of water results from tides, winds, currents and storms.

4) Thermal noise: It is taken as additive white Gaussian noise. Additive white Gaussian noise is the noise model used to mimic the effect of many random processes that occur in nature. Thermal noise is created by molecular agitation at the receiver side and it is always present in communication system. The Thermal noise formulated as

$10 \log N_{t h}(f)=-15+20 \log f$

5) Total noise: The overall noise power spectral density for a given frequency $f$ can be computed by adding all types of noise as [16]

$N(f)=N_{t}(f)+N_{s}(f)+N_{\omega}(f)+N_{t h}(f)$ [18].

These noise effects are modeled by Gaussian statistics

\section{Signal-to-Noise Ratio}

The SNR is attenuation that arises by the noise and frequency. Its power spectral density declines by frequency and SNR which differs over bandwidth of signal. If there are frequencies with narrow band having width as $\Delta \mathrm{f}$ and $f$ as frequency then SNR is modeled by
$\operatorname{SNR}(l, f)=\frac{S_{l}(f) \triangle f}{A(l, f) N(f) \triangle f}$

where PSD of transferred signal is $S_{l}(f)$. Thus the narrowband SNR is a frequency function for a specific distance. To attain a pre specified SNR, required power is expressed as $P(l)=p \times l^{\psi}$ and bandwidth $(l)=b \times l^{-\beta}$.

where the exponents $\beta \in(0,1), \psi \geq 1$, and $\mathrm{b}, \mathrm{p}$ coefficients based on target SNR, ambient noise and acoustic path-loss parameters [20].

\section{Bandwidth}

Bandwidth $B$ is the acoustic channel capacity that is on the order of center frequency expressed as $f_{c}$. It impacts on design of signal processing procedures, as it prevents one from making the narrowband assumption, $B \ll f_{c}$. Since multihopping confirms lesser energy consumption, its outcomes doubled for acoustic channel in perspective of energy-per-bit consumption [20]. The interference and collision increased as hops raises hence more retransmissions are required while shorter hops provide greater bit rates and the collision is mostly minimized. The channel capacity is sternly restricted at longer distances: at 100 kilo meter, just a $\mathrm{kHz}$ approximately is present. For the smaller range, the capacity rises, but it will eventually be restricted by the transducer. The fact that this channel capacity is restricted denotes the requisite for bandwidth efficient modulation methods in case greater than $\mathrm{bps} / \mathrm{Hz}$ is to be attained over these links.

\section{E. Multi Path Propagation}

Multi path establishment in water is directed by two major effects: first one is sound reflection at surface, bottom and any objects, and sound refraction in water. The later one is a significance of speed of sound variation with the depth that is typically evident in deep water links.

The sound speed depends on temperature and pressure that differ by the water depth. According to the Snell's law, sound wave must movess towards lower propagation speed area. The pressure and temperature are fixed in shallow water. The temperature decline as the depth rises. The mass of water above is insignificant change the pressure. The speed of sound reduces in the area known as main thermocline. The temperature reaches at $4^{\circ} \mathrm{C}$ after a little depth, and from there on, speed of sound rises with pressure. When the sound rays initiates, every ray follows a little diverse route and the receiver receives these multiple signals. For putting a mathematical modeling of channel in a view let nominal length represent using $\bar{l}_{p}$ of the $\mathrm{p}^{\text {th }}$ propagation path, where $\mathrm{p}$ $=0$.

The path delays acquired is $\bar{\tau}_{p}=\bar{l}_{p} / c$ where $c$, speed of sound taken constant in the shallow water.

Under an ideal situation the coefficient of surface reflection is (-1). Depending on the hard or soft bottom and grazing angle the coefficient of bottom reflection is different. [21].

If the cumulative reflection coefficient we represent by $\Gamma_{p}$ with propagation path $\mathrm{p}^{\text {th }}$ and the propagation loss by $A\left(\bar{l}_{p}, f\right)$ associated with this path, then 
$\bar{H}_{p}(f)=\frac{\Gamma_{p}}{\sqrt{A\left(\bar{l}_{p}, f\right)}}$

It denotes p-th path nominal frequency response and all paths perform as low pass filter. The coefficient of absorption do not vary with its value at referenced center frequency and distance hence for interested frequencies and the distances to the mainstream acoustic transmission systems, impact of the path filtering is about similar for entire channel. Signifying this value using $a_{0}$, function of transfer of $\mathrm{p}^{\text {th }}$ link is expressed as

$\bar{H}_{p}(f, t)=\bar{h}_{p} \quad$ x $\quad \bar{H}_{0}(f)$

$\bar{h}_{p}=\frac{\Gamma_{p}}{\sqrt{\left(\bar{l}_{p} / \bar{l}_{0}\right)^{k} a_{0}^{\bar{l}_{p}-\bar{l}_{0}}}}$

\section{F. Delay Spread}

The $T_{m p}$ 'represents the channel's overall delay spread. It is a vital characteristic to model a system of acoustic transmission. The small multi-path spreads revealed by perpendicular channels and large spreads which is possible up to 100's of mille seconds, by horizontal links.

In broadband systems that are single carrier having short data symbols by comparison with multipath spread, amount of symbols is of significant quality that specifies the Inter Symbol Interference (ISI) degree. It edicts filters size that requires to balance the link. The coherence of frequency which is an expressive quality for broadband systems represent by $\triangle f_{\text {coh }}$ which is equal to the $1 / T_{m p}$. According to multicarrier systems, less than this value parting of carrier is well preserved. It is also important to keep in mind that the channels of acoustic wave are sparse a lot.

$L \sim T_{m p} B$ is the total multipath spread with intervals $T_{s}=1 / B$ which is less than the $L$ coefficients that suit for signifying retort of channel $[22,23]$.

\section{G. Doppler Distortion}

Time variability of the channel is due to the two reasons, First one is the inherent variations in the propagation channel that results to fading of signal. And second one arises due to the sender and receiver mobility that results in frequency shifting. The difference between these two is that the former occurs randomly and second one is expressed in a deterministic way. Motion of sender and receiver or point of reflection with path of signal reasons the distances of path to change with time. The Doppler Effect magnitude is directly proportional to ratio $a=v / c$ of relative sender and receiver velocity to the sound speed.

As the speed of electro-magnetic waves is greater than the sound speed hence acoustic signal's, motion induced Doppler distortion is very high.

For the modeling of the Doppler distortion, let we take only propagation path and assumes the relative sender and receiver velocity $v_{p}$ that remains constant with this path for some interval of time then path delay expressed as

$\bar{\tau}_{p}(t)=\bar{\tau}_{p}-a_{p} \times t$ where the Doppler factor for the $\mathrm{p}^{\text {th }}$ path is $a_{p}=v_{p} / c$.

These factors about equal for entire paths $a_{p}=a, \forall p$, if we have only one dominant component of velocity[23].

To measure time variation delay effect for a signal, suppose we just emphasis at a specific component of the signal. It is in narrowband centered around frequency $f_{k}$ such that $\Delta f \ll f_{k}$. Following is relation of signal that is $s_{k}(t)$ with corresponding baseband $u_{k}(t)$ are given

$s_{k}(t)=\operatorname{Re}\left\{u_{k}(t) e^{j 2 \pi f_{k} t}\right\}$

As signal occupies frequencies narrow band, its copy is received over propagation path $\mathrm{p}^{\text {th }}$ that is given as

$s_{k, p}(t)=\bar{h}_{p} Q\left(f_{k}\right) s_{k}\left(t-\bar{\tau}_{p}(t)\right)$

The equivalent baseband component of the received signal through

$s_{k, p}(t)=R e\left\{v_{k, p}(t) e^{j 2 \pi f_{k} t}\right\}$

The association of baseband

$v_{k, p}(t)=\bar{c}_{k, p} e^{j 2 \pi a_{p} f_{k} t} u_{k}\left(t+a_{p} t-\bar{\tau}_{p}\right)$

where $\bar{c}_{k, p}$ is constant.

$\bar{c}_{k, p}=\bar{h}_{p} \bar{H}_{0}\left(f_{k}\right) e^{-j 2 \pi f_{k} \tau_{p}}$

and the Doppler effect are apparent in two factors. First is frequency shifting by the $a_{p} f_{k}$ while the other is time scaling by the factor $\left(1+a_{p}\right)$.

\section{H. Fading}

The inherent channel varies from very large scale, the slow changes which happens on season base such as the variation in speed of sound profile from summer to winter or everyday such as by the water currents depth variations, to minor scale, the rapid changes which produced by the quick mobility of the water surface.

The large scale occurrences affect ordinary signal power, producing it to change over lengthier time. The small scale occurrences upset rapidly the level of signal, producing change for smaller time.

1) Large-scale effects: Length of the path diverge from the nominal values for measuring arbitrary channel variations, hence.

$l_{p}=\bar{l}_{p}+\triangle l_{p}$

In equation (19) random displacement is denoted by $\Delta l_{p}$, that designs variations of large scale. The link transfer function that is now random is expressed by

$H(f)=\bar{H}_{0}(f) \sum_{p} h_{p} e^{-j 2 \pi f \tau_{p}}$

where $h_{p}$ is the path coefficients which is computed same as the nominal equation (12), by $l_{p}$ instead of $\bar{l}_{p}$ while delays can be computed as $\tau_{p}=l_{p} / c-t_{0}$ in reference to some time, such as. $t_{0}=\bar{l}_{0} / c$. The corresponding arbitrary path gains also estimated as [24] 
$h_{p}=\bar{h}_{p} e^{-\xi \Delta l_{p} / 2}$

$\xi_{p}=a_{c}-1+k / \bar{l}_{p}$

$\bar{h}_{p}$ is calculated by equation (12).

2) Small-scale effects: Previous model does not comprises impacts of scattering that produces dispersion of micro path with every propagation-path. It is shown by a factor which attends path gain $h_{p}(t)$. A complete model of channel is acquired as

$H(f, t)=\bar{H}_{0}(f) \sum_{p} \gamma_{p}(f, t) h_{p}(t) e^{-j 2 \pi f \tau_{p(t)}}$

In this model, the factor $h_{p}(t)$ represents slower changing process. The factor $\gamma_{p}(f, t)$ denotes faster changing scattering process. These factors are often expressed as circularly symmetric complex valued Gaussian processes which are normalized so as not to modify total signal power that is received, i.e.

$E\left\{\left|\gamma_{p}(f, t)\right|^{2}\right\}=1$

\section{CONCLUSION AND FUTURE WORK}

The channel modeling becomes significant aspect of signal processing and analyzing has been examined for decision feedback equalization [22, 23], turbo equalization [24, 25] and multi carrier revealing [26, 27, 28]. Though a larger channel capacity indicates more ISI and frequency discrimination, it infers an enhanced resolution in delay. Hence, the associated signal distortion is apparent as more simple, channel estimation is additionally effective in case when an appropriate sparse model is used, and that leads to better processing of signal. Moreover the signal at a greater rate allows more recurrent channel interpretations thus channel tracking will be easier [29]. Particularly, outstanding efforts are proceeding for merging the small range with greater bit rate UW acoustic transmission systems along leisurelier and lengthier range UW communications [30].

\section{REFERENCES}

[1] Stojanovic, Milica, and James Preisig. "Underwater acoustic communication channels: Propagation models and statistical characterization." IEEE Communications Magazine 47, no. 1 (2009): 84-89.

[2] Harris III, Albert F., and Michele Zorzi. "Modeling the underwater acoustic channel in ns2." In Proceedings of the 2nd international conference on Performance evaluation methodologies and tools, p. 18. ICST (Institute for Computer Sciences, Social-Informatics and Telecommunications Engineering), 2007.

[3] Akyildiz, Ian F., Dario Pompili, and Tommaso Melodia. "Underwater acoustic sensor networks: research challenges." Ad hoc networks 3, no. 3 (2005): 257-279.

[4] X. Che, I. Wells, G. Dickers, P. Kear, and X. Gong, "Re-evaluation of rf electromagnetic communication in underwater sensor networks," Communications Magazine, IEEE, vol. 48, no. 12, pp. 143 -151, Dec 2010.

[5] N. Farr, A. Bowen, J. Ware, C. Pontbriand, and M. Tivey, "An integrated, underwater optical /acoustic communications system," in OCEANS 2010 IEEE - Sydney, may 2010, pp. 1 -6.

[6] L. Freitag and S. Singh, "Performance of micro-modem psk signaling under variable conditions during the 2008 race and space experiments," in OCEANS 2009, MTS/IEEE Biloxi - Marine Technology for Our Future: Global and Local Challenges, october 2009, pp. $1-8$.
[7] L. Freitag and M. Stojanovic, "Basin-scale acoustic communication: a feasibility study using tomography m-sequences," in OCEANS, 2001. MTS/IEEE Conference and Exhibition, vol. 4, 2001, pp. $2256-2261$ vol.4.

[8] L. Freitag, M. Johnson, and D. Frye, "High-rate acoustic communications for ocean observatories-performance testing over a $3000 \mathrm{~m}$ vertical path," in OCEANS 2000 IEEE Conference and Exhibition, vol. 2, 2000.

[9] T. Curtin, J. Bellingham, J. Catipovic, and D. Webb, "Autonomous oceanographic sampling networks," Oceanography, vol. 6, no. 3, pp. 86 $-94,1993$.

[10] J. Potter, A. Berni, J. Alves, D. Merani, G. Zappa, and R. Been, "Underwater communications protocols and architecture developments at nurc," in OCEANS, 2011 IEEE - Spain, june 2011, pp. 1 -6.

[11] A. Quazi and W. Konrad, "Underwater acoustic communications," Communications Magazine, IEEE, vol. 20, no. 2, pp. 24 -30, march 1982.

[12] A. Baggeroer, D. Koelsch, K. von der Heydt, and J. Catipovic, "Dats - a digital acoustic telemetry system for underwater communications," in OCEANS 81, sep 1981.

[13] J. Catipovic, D. Brady, and S. Etchemendy, "Development of underwater acoustic modems and networks," Oceanography, vol. 6, no. $3,1993$.

[14] M. Green and J. Rice, "Channel-tolerant fh-mfsk acoustic signaling for undersea communications and networks," Oceanic Engineering, IEEE Journal of, vol. 25, no. 1, pp. $28-39$, january 2000.

[15] M. Stojanovic, J. Catipovic, and J. Proakis, "Phase-coherent digital communications for underwater acoustic channels," Oceanic Engineering, IEEE Journal of, vol. 19, no. 1, pp. 100-111, Jan 1994.

[16] Kularia, Yamini, Sheena Kohli, and Partha Pratim Bhattacharya. "Analysis Of Acoustic Channel Characteristics For Underwater Wireless Sensor Networks." International Journal of Computational Science, Information Technology and Control Engineering Vol.3, No.1/2, April 2016.

[17] Ahmed, S., N. Javaid, F. A. Khan, M. Y. Durrani, A. Ali, A. Shaukat, M. M. Sandhu, Z. A. Khan, and U. Qasim. "Co-UWSN: cooperative energy-efficient protocol for underwater WSNs." International Journal of Distributed Sensor Networks 2015 (2015): 75.

[18] Ahmed, Sheeraz, Nadeem Javaid, Ashfaq Ahmad, Imran Ahmed, Mehr Yahya Durrani, Armughan Ali, Syed Bilal Haider, and Manzoor Ilahi. "SPARCO: Stochastic Performance Analysis with Reliability and Cooperation for Underwater Wireless Sensor Networks." Journal of Sensors2016 (2016).

[19] M. Stojanovic, "On the relationship between capacity and distance in an underwater acoustic communication channel," in ACM SIGMOBILE Mobile Computing and Communications Review (MC2R). New York, NY, USA: ACM, 2007, pp. 34-43.

[20] "Capacity of a relay acoustic channel," Oceans 2007, pp. 1-7, Oct. 2007.

[21] M. Stojanovic, "Efficient processing of acoustic signals for high rate information transmission over sparse underwater channels," Elsevier Journal on Physical Communication, pp. 146-161, june 2008.

[22] W. Li and J. Preisig, "Estimation of rapidly time-varying sparse channels," Oceanic Engineering, IEEE Journal of, vol. 32, no. 4, pp. 927 -939, october 2007.

[23] S. Roy, T. Duman, and V. McDonald, "Error rate improvement in underwater mimo communications using sparse partial response equalization," Oceanic Engineering, IEEE Journal of, vol. 34, no. 2, pp. $181-201$, april 2009.

[24] W. Yang and T. Yang, "High-frequency channel characterization for mary frequency-shift-keying underwater acoustic communications," Journal of the Acoustical Society of America, vol. 120, no. 5, pp. 26152626, november 2006.

[25] B. Tomasi, G. Zappa, K. McCoy, P. Casari, and M. Zorzi, "Experimental study of the space-time properties of acoustic channels for underwater communications," in OCEANS 2010 IEEE - Sydney, may 2010, pp. $1-9$. 
[26] A. Radosevic, D. Fertonani, T. Duman, J. Proakis, and M. Stojanovic, "Capacity of mimo systems in shallow water acoustic channels," in Signals, Systems and Computers (ASILOMAR), 2010 Conference Record of the Forty Fourth Asilomar Conference on, nov. 2010.

[27] F. Socheleau, C. Laot, and J. Passerieux, "Stochastic replay of nonwssus underwater acoustic communication channels recorded at sea," Signal Processing, IEEE Transactions on, vol. 59, no. 10, oct. 2011.

[28] M. Chitre, "A high-frequency warm shallow water acoustic communications channel model and measurements," Journal of the
Acoustical Society of America, vol. 122, no. 5, pp. 2580-2586, november 2007.

[29] J. Zhang, J. Cross, and Y. Zheng, "Statistical channel modeling of wireless shallow water acoustic communications from experiment data," in Military Communications Conference, 2010 - MILCOM 2010, 31 2010-nov. 3 2010, pp. $2412-2416$.

[30] J. W. Choi, T. Riedl, K. Kim, A. Singer, and J. Preisig, "Adaptive linear turbo equalization over doubly selective channels," Oceanic Engineering, IEEE Journal of, vol. 36, no. 4, pp. $473-489$, october 2011. 